\title{
Molecular Structure Characterization of the Extracts from Soybean Dreg Guizhen Gong ${ }^{\mathrm{a}^{\star}}$ and Congyan Sun ${ }^{\mathrm{b}}$

\author{
School of Chemical Engineering, Xuzhou University of technology, Xuzhou 221018, China
} \\ aggz72@163.com, b1325233807@qq.com
}

\section{Keywords: Soybean dreg; Extract; Acetone; GC/MS Aanalysis}

\begin{abstract}
Soybean dreg was extracted with acetone in Soxhlet Extractor. The extracts were analyzed with GC/MS. In total, 53 kinds of organic compounds were detected in extracts, which can be divided into seven classes according to the difference of molecular structure and functional group: alkanes (As), unsaturated hydrocarbonaromatic (UHs), aromatic hydrocarbons (Ars), aldehydes (Als), acids, esters (Es) and other species (OS), respectively. The relative content (RC) of the above species decrease in the order: Als $>>$ acids $>$ Es $>$ OS $>$ Ars $>$ UHs $>$ As. Als are the most abundant, accounting for $51.4 \%$. The study has important significance basic theory on the development of bean dreg with high added value utilization.
\end{abstract}

\section{Introduction}

Soybean dreg, as by-product of soybean processing industry, is about $16 \% \sim 25 \%$ of the whole soybean quality [1]. In China, nearly 20 million tons of soybean dreg as residue are produced every year [2]. According to the analysis, there are rich nutrients and physiological functions in soybean dreg [3-6]. Soybean dreg is susceptible to corruption due to the high content of the water, so soybean dreg is usually treated as a waste. At present, only a small part of the soybean dreg is consumed in a traditional way, such as animal feed, food production and extraction of functional components [7]. Until recently, the majority of soybean dreg has not been used and disposed off, causing environmental and public health problems [8]. Therefore, efficient utilization of which draws increasing attention in China.

In the present study, soybean dreg was extracted with acetone in a modified Soxhlet extractor. The extracts were analyzed with GC/MS and FTIR.

\section{Experimental}

Materials. Fresh wet soybean dreg was purchased from Xuzhou farmer market, in Jiangsu, China, which was dried in a vacuum at $65{ }^{\circ} \mathrm{C}$ for $24 \mathrm{~h}$, and then cooled to room temperature under keeping vacuum state. The soybean dreg dried was pulverized to pass through a 150-mesh sieve, and kept in dryer. Acetone is commercially purchased analytical one and purified by distillation prior to use 。

Methods. $5 \mathrm{~g}$ soybean and $120 \mathrm{~mL}$ acetone were put into Soxhlet extractor. The Soxhlet extractor was heated up to $56{ }^{\circ} \mathrm{C}$ and kept at the temperature for $5 \mathrm{~h}$, then cooled down to room temperature. The extracts mixture was taken out from the extractor. The extract was distilled using a Büchi R-134 rotary evaporator to afford acetone extract (AE), which was analyzed with GC/MS.

\section{Results and Discussion}

GC/MS Analysis. Fig.1 show the total ion chromatograms (TICs) of extracts. The corresponding compounds identified are listed in Tables 1 to 5 . In total, 53 kinds of organic compounds were detected in extracts, which can be divided into seven classes according to the difference of molecular structure and functional group: alkanes (As), unsaturated hydrocarbonaromatic (UHs), aromatic hydrocarbons (Ars), aldehydes (Als), acids, esters (Es) and other species (OS), respectively. The relative content $(\mathrm{RC})$ of the above species is illustrated in fig. 2. As this figure shows, the yields of 
products decrease in the order: Als $>>$ acids $>$ Es $>\mathrm{OS}>$ Ars $>\mathrm{UHs}>$ As, and Als are the most abundant, accounting for $51.4 \%$, indicating Als can be enriched by acetone from soybean dreg.

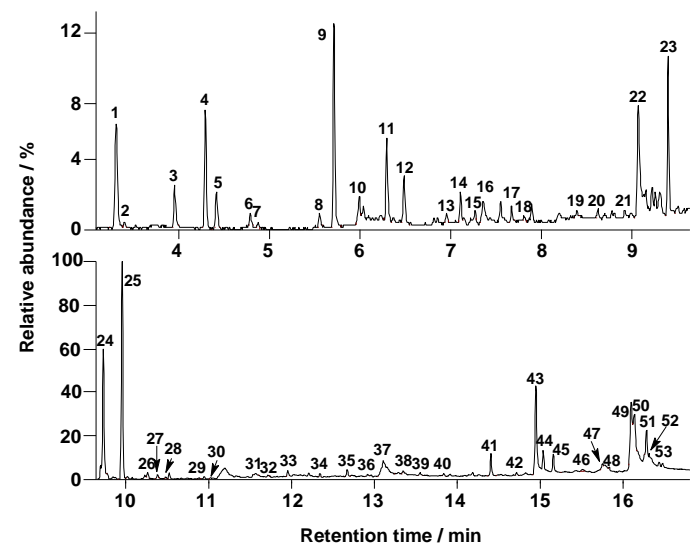

Figure 1. TIC of AE from soybean dreg.

Table 1 Alkanes and aromatic hydrocarbons identified in AE from soybean dreg

\begin{tabular}{|c|l|c|c|}
\hline Peak & \multicolumn{1}{|c|}{ Compounds } & Peak & Compounds \\
\hline & \multicolumn{1}{|c|}{ Aromatic hydrocarbons } & & Alkanes \\
\hline 4 & Ortho xylene & 34 & hexadecane \\
\hline 5 & p-xylene & 40 & octadecane \\
\hline 6 & Meta xylene & 46 & 1-chlorooctadecane \\
\hline 29 & 1,5-dimethylnaphthalene & & \\
\hline 30 & 1,6-dimethylnaphthalene & & \\
\hline
\end{tabular}

Table 2 Unsaturated hydrocarbon identified in AE from soybean dreg

\begin{tabular}{|c|l|c|l|}
\hline Peak & \multicolumn{1}{|c|}{ Compounds } & Peak & \multicolumn{1}{|c|}{ Compounds } \\
\hline 2 & (E)-oct-2-ene & 26 & octadec-1-yne \\
\hline 8 & (E)-2,4,6-trimethylhept-3-ene & 27 & tridec-1-ene \\
\hline 13 & (E)-undec-1-ene & 47 & henicos-1-ene \\
\hline 20 & (E)-dodec-3-ene & & \\
\hline
\end{tabular}

Table 3 Aldehyde identified in AE from soybean dreg

\begin{tabular}{|c|l|c|l|}
\hline Peak & \multicolumn{1}{|c|}{ Compounds } & Peak & \multicolumn{1}{c|}{ Compounds } \\
\hline 1 & (E)-hex-2-enal & 21 & (2E,4E)-nona-2,4-dienal \\
\hline 9 & (E)-hept-2-enal & 22 & 5-(hydroxymethyl)furan-2-carbaldehyde \\
\hline 11 & (2E,4E)-hepta-2,4-dienal & 23 & (Z)-dec-2-enal \\
\hline 12 & (2Z,4Z)-hepta-2,4-dienal & 24 & (2E,4E)-deca-2,4-dienal \\
\hline 14 & (E)-oct-2-enal & 25 & (2Z,4Z)-deca-2,4-dienal \\
\hline 17 & nonanal & 36 & (10Z,12Z)-hexadeca-10,12-dienal \\
\hline
\end{tabular}


Table 4 Acids and Ester identified in AE from soybean dreg

\begin{tabular}{|c|l|c|l|}
\hline Peak & \multicolumn{1}{|c|}{ Acids } & Peak & \multicolumn{1}{|c|}{ Ester } \\
\hline 10 & Hexanoic acid & 28 & dl-3,4-Dehydroproline methyl ester \\
\hline 19 & Octanoic Acid & 41 & diisobutyl phthalate \\
\hline 31 & 11-bromoundecanoic acid & 42 & methyl palmitate \\
\hline 39 & tetradecanoic acid & 44 & dibutyl phthalate \\
\hline 43 & palmitic acid & 45 & ethyl palmitate \\
\hline 49 & (9Z,12Z)-octadeca-9,12-dienoic & 48 & (9Z,12Z)-methyl octadeca-9,12-dienoate \\
\hline 52 & oleic acid & 51 & (9Z,12Z)-ethyl octadeca-9,12-dienoate \\
\hline
\end{tabular}

Table 5 Other substances identified in AE from soybean dreg

\begin{tabular}{|c|l|c|l|}
\hline Peak & \multicolumn{1}{|c|}{ Compounds } & Peak & \multicolumn{1}{|c|}{ Compounds } \\
\hline 3 & 2-methylhexan-2-ol & 33 & $\begin{array}{l}\text { N-(4-chlorophenyl)-3-ethoxythiophene-2- } \\
\text { carboxamide }\end{array}$ \\
\hline 7 & dodec-11-en-2-one & 35 & diphenylamine \\
\hline 15 & (3E,5E)-octa-3,5-dien-2-one & 37 & 4,6-Di-O-methyl-.alpha.-d-galactose \\
\hline 16 & $\begin{array}{l}\text { 6-butyl-3-methoxycyclohex-2- } \\
\text { enone }\end{array}$ & 38 & 2-hexyldecan-1-ol \\
\hline 18 & Maltol & 50 & N-(2-hydroxyethyl)palmitamide \\
\hline 32 & 2-amino-4,6-dimethylphenol & 53 & (E)-octadec-9-enamide \\
\hline
\end{tabular}

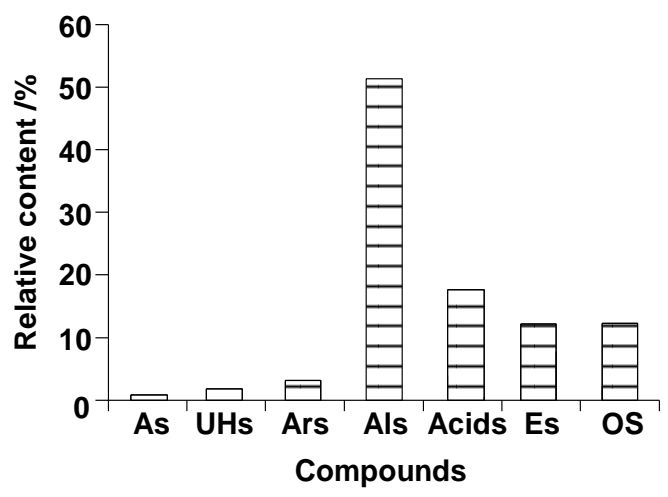

Figure 2. Distribution of different types of products in AE from bean dreg

As is shown in fig. 2, 3 As were detected with $0.9 \%$ of relative content, including hexadecane (peak 34), octadecane (peak 40), and 1-chlorooctadecane (peak 46). They may originally be present in wax layer of soybean. The Ars detected include 3 xylenes (peaks 4, 5, and 6), and 2 dimethylnaphthalene (peaks 29, and 30), with $3.3 \%$ of relative content. 7 UHs were identified as exhibited in table 2. Among the UHs, octadec-1-yne and henicos-1-ene is predominantly abundant.

These hydrocarbons are value-added chemicals in the application of many aspects, especially as raw materials for manufacturing chlorinated paraffin, senior spices, plastic, dyestuff, medicine and cosmetic. At present, they are mainly derived from fossil resources, such as oil, natural gas and coal. With the reduction of fossil resource reserves, the price will be affected. Therefore, further enriching and subsequently purifying the hydrocarbons is an important subject. 
As were the most detected species with $51.4 \%$ of relative content in soybean dreg by GC/MS. As listed in Table 3, in total 12 As were identified in the products. Most of these As were unsaturated aldehydes, including 4 enal (peak 1, 9, 14, 23 in Fig. 2) and 6 dienal (peak 11,12, 21, 24,25, 36 in Fig. $2)$. The yields of $(2 \mathrm{E}, 4 \mathrm{E})$-deca-2,4-dienal and $(2 \mathrm{Z}, 4 \mathrm{Z})$-deca-2,4-dienal are much higher than other As, accounting for $14.2 \%$ and $22.7 \%$, respectively. Enal, with special aroma, plays an important role in the flavor industry, such as (2E,4E)-hepta-2,4-dienal and (2E,4E)-deca-2,4-dienal.

As are mainly obtained from synthesized. If As can be isolated from soybean dreg will help reduce the material price, while improving the utilization value of soybean dreg.

As listed in Tables 4, 7 acids and 7 esters were detected in total. All of the acids are fatty acids accounting for $17.7 \%$, consisting of 2 unsaturated fatty acids and 5 saturated fatty acids. Ester detected contained 2 phthalate esters and 5 aliphatic ester. These compounds have very important practical value at many fields such as Paints, adhesives, dyes, pesticides, spices, etc.

There are 12 OSs detected with $12.3 \%$ of RC, including 2 alcohols (peak 3, 38 in table 5), 3 ketones (peak 7, 15,16 in table 5), 2 phenols (peak 18, 32 in table 5), 1 sugar(peak 37 in table 5), and 4 nitrogen containing compounds.

\section{Summary}

Soybean dreg was extracted with acetone in Soxhlet Extractor. A series of As, UHs, Ars, Als, acids, Es and OS were successively enriched, The relative content (RC) of the above species decrease in the order: Als $>>$ acids $>$ Es $>$ OS $>$ Ars $>$ UHs $>$ As. Als are the most abundant, accounting for $51.4 \%$. The study has important significance basic theory on the development of bean dreg with high added value utilization.

\section{Acknowledgement}

This work was supported by the China Building Material Federation (2014-M3-4) and Xuzhou Information Institute (XKQ016).

\section{References}

[1] Y.X. Sun, X.Y. Wu, Y.H. Wang, Y. Luo, B.C. Liu and W.J. Xu, Study on preparing watersoluble dietary fiber from soybean residue, Food and Fermentation Industries, vol. 35 (2009), pp. 92-95.

[2] Y. Chen, R. Ye, L. Yin and N. Zhang, Novel blasting extrusion processing improved the physicochemical properties of soluble dietary fiber from soybean residue and in vivo evaluation, J. Food Eng. vol. 120 (2014), pp. 1-8.

[3] E. M. He, H. H. Li, Q. Chang, W. wang and J. Xu. A Preliminary study on extraction of soybean isoflavones from soybean dregs by ultrasound method, Soybean Science, vol. 30 (2011), pp. 680-682.

[4] Y. Yin, X. F. Li, G. H. Song, Q. Yue, X. Y. Zeng and S. G. Liu. Ultrasonic extraction technology of soluble soybean polysaccharides, J. Hui Zhou University, vol. 30 (2010), pp. 50-53.

[5] C. Y. Ma, W. S. Liu, K. C. Kwok and F. Kwok. Isolation and characterization of proteins from soymilk residue, Food Research International, vol. 29 (1997), pp. 799-805.

[6] Y. J. Fan, Q. Zhang and B. Zhu. Study on extraction of soluble soybean polysaccharides, Food Science, vol. 28 (2007), pp. 295-298.

[7] A. Redondo-cuenca, M. A. Villanuva-suamajose and I. Mateos-aparicio. Soybean seeds and its by-product okara as sources of dietary fibre. measurement by AOAC and Englyst methods, Food Chem., vol. 108 (2008), pp. 1099-1105.

[8] D. K. O' Toole. Characteristics and use of Okara, the soybean residue from soy milk production- a review, J. Agric. Food. Chem., vol. 47 (1999), pp. 363 - 371. 